\title{
Mamás psicoanalíticas y bebés psicoanalíticos: Una visión ampliada ${ }^{1}$
}

\author{
Joyce Slochower, Ph.D., ABPP 2 \\ Programa Postdoctoral de la Universidad de Nueva York
}

\begin{abstract}
Ofrezco una visión en retrospectiva de la metáfora del bebé en desarrollo en el pensamiento relacional. Las primeras críticas relacionales al enfoque del desarrollo y el concepto de contención (holding) en el trabajo clínico, amplificado por escritoras feministas, hizo que los relacionales dieran un fuerte giro hacia una visión del paciente-como-adulto, y una visión del dialogo analítico como algo inherentemente intersubjetivo. Al traer mi propia perspectiva Winnicottiana / relacional a esta crítica, expando la noción de la contención/sostén y propongo un puente entre los dos modelos, explorando la participación del analista para establecer y mantener la experiencia de contención/sostén. Aquí, ofrezco un resumen de esta controversia y su evolución/resolución, detallando cómo ha cambiado mi propia comprensión de la metáfora de la contención y la dinámica subyacente. Ilustro las múltiples encarnaciones de la función de contención con casos viñeta cortos y más largos.
\end{abstract}

Palabras clave: Sostenimiento, Desarrollo psíquico, Winnicott, Psicoanálisis Relacional.

I offer a retrospective view of the evolving baby metaphor in relational thinking. Early relational critiques of the developmental tilt and the concept of holding in clinical work, amplified by feminist writers, sharply skewed relationalists toward a vision of the patient-as-adult and a view of the analytic dialogue as inherently intersubjective. Bringing my own Winnicottian/relational perspective to this critique, l expanded the notion of holding and proposed a way to bridge the two models by exploring the analyst's participation in establishing and maintaining a holding experience. Here, I offer an overview of this controversy and its evolution/resolution, detailing how my own understanding of the holding metaphor and its underlying dynamics has changed. I illustrate holding's multiple incarnations with short and longer case vignettes.

Key Words: Holding, Psychological Development, Winnicott, Relational Psychoanalysis.

English Title: Psychoanalytic Mommies and Psychoanalytic Babies: A Long view.

\section{Cita bibliográfica / Reference citation:}

Slochower, J. (2018). Mamás psicoanalíticas y bebés psicoanalíticos: Una visión ampliada. Clínica e Investigación Relacional, 12 (3): 444-464. [ISSN 1988-2939] [Recuperado de www.ceir.info ] DOI: 10.21110/19882939.2018.120303

\footnotetext{
${ }^{1}$ Texto de la intervención de Joyce Slochower en ÁGORA RELACIONAL (Madrid) en el Seminario Clínico del 30 de Junio de 2018. Basado en una Presentación Plenaria en la reunión Anual 2012 IARPP, Nueva York. La autora expresa sus agradecimientos a Jim Anderson, Tony Bass, Sue Grand, Margery Kalb y Don Stern por sus atentos comentarios al borrador anterior de este trabajo. Partes de este trabajo se han publicado como: Slochower, J. (2013). Psychoanalytic Mommies and Psychoanalytic Babies: A Long View. Contemp. Psychoanal., 49(4):606-628. Reproducido aquí el texto de su intervención en Madrid, con autorización de la autora. Traducción al castellano de María Hernández Gázquez.

2 Joyce Slochower es Doctora en Psicología y Psicoanalista (individual y de parejas); vive y trabaja en Nueva York. Profesora y supervisora en el Programa Postdoctoral de la Universidad de Nueva York, del Steven Mitchell Center for Relational Psychoanalysis entre otros. Entre sus publicaciones Excessive Eating: The Role of Emotions and Environment (1983), Holding and Psychoanalysis: A Relational Approach (1996) y Psychoanalytic Collisions (2006). Contacto: 15 West 75 ${ }^{\text {th }}$ Street 8B - New York, N.Y. 10023 Joyce.slochower@gmail.com Tel. 212-362-4437 CelR Vol. 12 (3) - Octubre 2018 ISSN 1988-2939 - www.ceir.info

(c) Derechos reservados/Copyright de Clínica e investigación Relacional y los autores. Prohibida la reproducción total o parcial sin autorización expresa. Este material es para uso científico y profesional exclusivamente y puede contener información clínica sensible. Los editores no se responsabilizan de los contenidos de los autores. Dirigir las consultas sobre derechos y autorizaciones a ceir@psicoterapiarelacional.es
} 
Crecí, hablando psicoanalíticamente, en los 80 . Al realizar el postgrado me presentaron las teorías de las relaciones de objeto y rápidamente me enamoré, especialmente de Winnicott. Las obras de Winnicott, poco convencionales a la vez que maternales, evocan una visión de responsividad afectiva, de unos padres nuevos y mejorados. Esa visión generó una poderosa respuesta. Si el analista simbólicamente puede ser la madre, la posibilidad de trabajar con traumas tempranos se incrementa enormemente; lo que no puede ser recordado puede ser re-experienciado y por tanto reparado; el paciente puede, de hecho, ser de nuevo un bebé, pero con una madre mejor y más responsiva.

No hay duda de que las fantasías - tanto inconscientes como explicitas- de una reparación parental están vivas en la consulta. La misma mañana que estaba escribiendo este trabajo, una paciente mujer de negocios que normalmente se precibe como enormemente adulta, y que siente que soy una asesora-par que la ayuda, se sentó y dijo, "Tengo que decirte que me dan ganas de hacerme una bola y llorar como una niña pequeña. He esperado tanto tiempo para llegar aquí. No me puedo creer que haya sido solo una semana y no me creo lo dependiente que me siento". No se quedó ahí, pero durante un momento conectó con un deseo de bebé y a medida que se hacía vivo afectivamente, resoné - tanto con su deseo como con la posibilidad de cumplirlo. Este fenómeno es tan común que incluso es corriente, aunque su lugar en nuestras teorías varia ampliamente.

Los pacientes, como bebés vulnerables y dependientes. No los de Freud organizados edípicamente, ni neuróticos en conflicto, ni los de Klein rabiosos y mordaces. De alguna forma, los de Ferenczi. Y por supuesto los del Grupo Independiente (Intermedio) Británico, quienes reconceptualizaron el proceso psicoanalítico como una reparación maternal simbólica para lo que ahora llamamos trauma relacional, invitando a la consulta el necesitado estado bebé - y la nutriente respuesta maternal. Aquí había un analista que podía enfrentarse a la necesidad, y permanecer estable y empático, aguantando el esfuerzo sin represalias.

Las metáforas sobre el desarrollo cambiaron el paisaje clínico al trasladar el trabajo clínico desde el enfoque en el conflicto sexual y agresivo (la relación repetida) enfocado hacia el "uno" necesitado (Steve Stern, 1994). Ese cambio no excluía la interpretación, pero dirigía esas interpretaciones hacia la vulnerabilidad. Las metáforas maternas dieron nombre y forma a algo que mayormente había permanecido sin articular - el valor clínico de la responsividad empática. Ahora se estaba teorizando: La dependencia no era defensiva, era real y requería una reparación real.

Por supuesto, esta perspectiva clínica no fue abrazada de forma universal. Parecía que bifurcaba el deseo y la necesidad, como si la reparación pudiera reemplazar el análisis de 
conflicto. ¿Dónde estaban, en esta perspectiva, las dinámicas de agresión y envidia - el apego a los objetos malos?

Otro tipo de crítica fue articulada por los escritores interpersonales y constructivistas sociales. Ellos - Mitchell (1984; 1988; 1993), Hoffman (1991), Don Stern (1992), Aron (1992) y otros rechazaban ambos lados de la metáfora maternal y las presunciones sobre las que se basaba. Esas presunciones - la certeza del analista, conocimiento y poder; de la posibilidad de delinear una 'verdad' histórica - se estaban retando de forma pronunciada: El analista relacional ni es omnisciente ni omnipotente; más aún, el paciente sabe más, y mucho más de lo que sabe un bebé. Lo que fue ya no es; el paciente trae su self no-bebé con todos sus conflictos relacionados y formas complejas de experimentar las cosas - a la consulta. Cuando se pone en escena, las ilusiones del desarrollo crean una situación terapéutica 'como si' que congela al paciente en una posición de dependencia desamparada mientras invoca la grandiosidad del analista.

Además de esta crítica surgieron voces enraizadas en el pensamiento feminista. Comenzando en los '6o, las feministas retaban tanto la idealización de la maternidad como la demanda asociada de auto-abnegación maternal, señalando que la visión tradicional de la maternidad obliteraba al padre y localizaba toda la patología infantil en el regazo materno. Esta posición excluía la idea, ni qué decir la experiencia, de la madre-comopersona.

Para mí, una joven madre luchando por equilibrar la carrera con la maternidad, la crítica feminista resonó en mí. Quería y sentía que tenía que hacerlo todo, y hacerlo fenomenalmente bien. Así que fue un alivio descubrir una grabación infantil del cantante americano Marlo Thomas titulado "Libres para ser tu y yo." Incluía una canción cuyo coro era "las mamás son personas, personas con niños". Finalmente, el sentido del reconocimiento (Todavía me sé toda la letra). Hoy creo que esto es escandaloso, lo escandalosas que eran esas palabras. Sin embargo, más de tres décadas después, de vez en cuando me resisto al impulso de cantárselo a mis hijos, ya adultos y casados. Aunque con el tiempo las madres se convierten en sujetos para sus hijos, es raro que un niño sustente ese conocimiento de forma continuada. $Y$ de muchas maneras, esto es también cierto para los pacientes vis a vis sus analistas.

Recogiendo y elaborando sobre este argumento, las psicoanalistas feministasDinnerstein (1976), Chodorow (1978), Fast (1984), Benjamin (1986; 1988), Harris (1991; 1997), Dimen (1991), Goldner (1991), Bassin (1997; 1999), Layton (1998) y otras (e.g. Bassin, Honey \& Kaplan, 1994; Kraemer, 1996) - recogieron el guante y lo introdujeron en la consulta, criticando las dicotómicas imágenes de género. Visiones de lo que Grand (2000) 
llama la abundancia materna, del analista-como-madre-tierra, niegan la naturaleza irreducible de la subjetividad analítica ${ }^{1}$ (Renik, 1993). E ignoran al padre pre-edípico.

Entonces, ¿hay o no un bebé en nuestra consulta? Y si lo hay, fue descubierta o fue creada¿por una analista cuyo sesgo teórico ofusca el actual? Los pensadores relacionales tempranos fueron claros: Ni hay bebe ni madre en la consulta. Únicamente hay dos adultos.

Es justo aquí cuando mi propio trabajo entró en escena. Influida por el movimiento feminista, Los Lazos de Amor (The Bonds of Love, 1988) de Benjamin y Sujetos Iguales, Objetos de Amor (Like Subjects, Love Objects,1995) - y por la tendencia critica al desarrollo, de Mitchell - propuse una alteración clínica/teórica a la metáfora de la contención que se pudiera incluir dentro de la teoría relacional, aunque fuera una forma expandida y complicada (Slochower, 1991; 1992; 1993; 1994; 1996a; 1996b; 1996c; 1999). Mitchell me invitó a publicar "La Contención ${ }^{2}$ y el psicoanálisis" en la Serie Relacional y así tuve el privilegio de escribir bajo su extraordinaria capacidad editora.

En mi obra "La contención y el Psicoanálisis" (Holding and Psychoanalysis) argumenté sobre el valor terapéutico de la responsividad empática, re-concebida para explicar las criticas relacionales y feministas y ampliada para abarcar una trayectoria del desarrollo que se extendiera más allá de la guardería. Aquí un breve resumen: El valor clínico del intercambio intersubjetivo tiene límites. Algunos pacientes especialmente vulnerables no pueden tolerar la alteridad del analista sin un descarrilamiento que en vez de abrir, cierra el proceso terapéutico. La contención permite la difuminación del limite permeable entre paciente y analista, amortiguando el impacto de la alteridad del analista, abriendo el proceso terapéutico.

Cuando trabajo dentro de la metáfora de la contención, encuentro que presto especial atención a las respuestas emocionales de mi paciente ante la evidencia de mi 'alteridad', es decir mis pensamientos, reacciones e ideas "separadas". No me refiero a si mi paciente acepta o no lo que digo; un enérgico "de ninguna manera jestás equivocada!" puede ser lo que abre un intercambio más rico y útil. Pero cuando mi paciente en estas ocasiones se cierra continuamente; cuando no es capaz de aceptar y trabajar con, o rechazar mi perspectiva mientras sostiene el suyo, me recolocó, hablando terapéuticamente. Me pregunto si quizás estaré 'equivocada', emocional o dinámicamente, si acaso estamos inmiscuidos en un potencialmente útil -o muy problemático- re-enactment. ¿Estará mi paciente reaccionando a que yo me esté pareciendo demasiado a los 'objetos viejos' o que esté siendo demasiado distinta a ellos?

Al no introducir mi 'alteridad' abiertamente en la consulta, ayudo a mi paciente a sentirse 'visto' no desde fuera hacia dentro, sino desde dentro hacia fuera, como describió 
Bromberg (1991). La contención puede representar un poderoso antídoto para las experiencias crónicas de haber sido aniquilado. No siempre se organiza en torno a la dependencia, pero puede ser una forma útil de trabajar con estados afectivos como la rabia, el desprecio, y similares. Aquí puedo contener intentando ser aceptada por el infractor (Davies \& Frawley, 1994; Grand, 2000; 2010), al luchar por sobrevivir y no contra-atacar (Winnicott, 1971). Este tipo de contención puede comunicar, vía la acción interpretativa (Ogden, 1994) que reconozco y acepto los sentimientos difíciles de mi paciente junto con una comunicación doble: me afectan, pero no me destruyen.

En ocasiones contener ayuda a mis pacientes a tomar un mayor contacto con emociones dolorosas agudas; en otros momentos, les ayuda a regular por disminución, saliendo de un estado emocional desbordante. Regular por disminución puede implicar la función de contención de Bion (1989) donde yo absorbo, metabolizo y reintroduzco estados afectivos tóxicos. Como alternativa, la regulación por disminución puede ocurrir a través de la danza de la díada interactiva que describen Beebe y Lachmann (2002).

Cualquier forma que tome, la metáfora de la contención nos lleva a apartar, parcialmente, la protesta parental/analítica (las mamas/analistas son personas). Por tanto, la contención requiere mucha auto-contención por parte del analista. $Y$ a pesar de lo que puedan pensar muchos críticos, contener a los pacientes normalmente no es algo divertido; puede ser opresivo, limitante, y dejarnos pensando que no estamos trabajando lo suficiente o que estamos continuamente aguantando la respiración, permaneciendo demasiado quietos, siguiendo a nuestro paciente demasiado de cerca. Contener el odio y el desprecio es aún menos divertido. Tal y como nos recuerdan Davies (2004), Epstein (1987) y otros, cuando somos el objeto malo para nuestro paciente, lo somos también para nosotros mismos. No sólo nuestros pacientes necesitan de la contención.

Invoco la idea de hacerle un paréntesis a la subjetividad para capturar el doble sentido de la experiencia de contención, la cualidad de estar-ahí-pero-casi-no-estar de mi subjetividad. Puedo sentirme estresada, cansada, impaciente, furiosa, en formas que pueden ser inquietantes, suscitando la ansiedad, o sino profundamente molestos para mi paciente. En esos momentos, hago un paréntesis tomando nota, y luchando con, pero intentando dejar de lado mi reacción sin desconocerla.

Es difícil describir todo esto sin que parezca muy deliberado, incluso coreografiado. Pero bajo mi punto de vista, el entrar y salir de la metáfora de la contención es todo menos eso: se determina por la multiplicidad, a vez consciente, intencional y no. En parte, me acerco a la contención basada en mi punto de entrada clínico/teórico. En parte este cambio es de procedimiento, una reacción espontánea a aspectos de mi propia experiencia que ni 
siquiera sé que estoy percibiendo. En parte, esta "enacted", responsivo a tira-y-aflojas de mi paciente que fueron responsivos a los tira-y-afloja que vienen de mí. Y para complicar las cosas más aun, algunas veces (todos) se fracasa cuando intentamos contener - porque creo que sé lo que necesita, pero no; porque estoy en medio de un enactment, un fracaso de auto-objeto, o algún otro tipo de no-sintonía. Es decir, hay claros límites a lo que puedo contener y a lo que puedo hacer paréntesis, porque no puedo hacer paréntesis de lo que no sé que estoy sintiendo. Otros pacientes, muchos de mis pacientes son muy perceptivos (a veces incluso más que yo), pueden recoger aspectos de mis reacciones a pesar de mis intentos de hacer un paréntesis. Así que para hacer un paréntesis se requiere a ambos; cuando mi paciente necesita no saber algo sobre mí, ella hace un paréntesis incluso más que yo, para protegerse de estos aspectos de mi alteridad (mi variabilidad, reactividad, etc.) que podrían romper o quebrar el sentido de resonancia de la que ella depende. El concepto de paréntesis mutuo extrae la contención del lado del analista y lo coloca en el espacio diádico, invirtiendo la asimetría asociada con la metáfora de la contención.

Un ejemplo muy dramático de este tipo de paréntesis me lleva a mis días como joven analista, cuando estaba con un embarazo muy avanzado de mi tercer hijo. Con 8 meses de embarazo, estaba enorme. Sintiendo que no podía seguir esperando a que mi paciente Jonathan, que ni estaba muy enfermo, ni especialmente disociado, afrontara lo obvio le dije, "tenemos que hablar sobre algo".

Esperando que reconociera que no había querido sacar a la luz mi embarazo, pero que, por supuesto, lo había notado, no anticipé que haría un quite y que literalmente se caería atrás en la silla, estupefacto.

La necesidad de Jonathan de vernos como una pareja dentro de este espacio protegido ofuscaba totalmente mi embarazo, una indicación muy concreta de mi alteridad. Había excluido mi embarazo, así como lo que representaba (la perspectiva de un hermano simbólico, ni qué decir de mi marido en la sombra - la pareja sexual oculta con quien había concebido este hijo). Al hacer esto, Jonathan había sostenido la experiencia esencial de unidad conmigo, la primera experiencia de este tipo que él recordaba. Sin embargo, nuestro espacio no era el de la contención reminiscente de la guardería. Jonathan me sentía más como un igual/hermana mayor que se identificaba con sus necesidades y era capaz de estar junto con él en esas necesidades. Un elemento de gemelaridad se fusionaba con sus deseos maternales de verme como 'una mujer, pero cómo él'. Por lo tanto, no embarazada. Y por mucho que yo conscientemente 'quisiera' ser vista en mi estado de embarazo, quizás a otro nivel inconscientemente estaba sustentando este paréntesis vía 
mi deseo de proteger nuestra relación (y a mi bebé), dejando esto último fuera del espacio terapéutico.

Con el tiempo Jonathan y yo hablamos sobre esto, sobre lo que él necesitaba evitar y por qué. Nuestras conversaciones fueron rellenando y dando cuerpo al dialogo terapéutico, pero estoy bastante segura de que no hubieran podido tener lugar si hubiera introducido insistentemente mi embarazo al principio. Merece destacar que nunca le dije a Jonathan que me había estado molestando que ignorara mi embarazo. Elegí no hacerlo ya que sentía que este tipo de auto-revelación hubiera avergonzado intensamente a este hombre tímido y sensible. Continuaré con este tema de nuevo más adelante.

\section{Falsas Dicotomías}

Ha habido una tendencia a posicionar la contención y la mutualidad como una polaridad caracterizada por la no-revelación en un extremo y auto-revelación total en el otro. Pero esta es una falsa dicotomía, y la mayoría de nosotros la hemos superado. A pesar de nuestros mejores intentos de contener, aspectos de nuestra persona - su capacidad y sus límites - se filtran al espacio terapéutico. Es inevitable; no podemos no mostrarnos. Además de lo cual, ya nos mostramos bastante en cómo y cuándo contenemos.

Pero el otro extremo de esta polaridad es igualmente elusiva: Aun cuando apuntamos hacia una total auto-revelación, para una mutua y continua exploración, nunca terminamos de llegar a ello. Ni, creo, debiéramos. La auto-revelación total es imposible (ya que como analistas tenemos nuestra propia experiencia inconsciente). Además no es deseable. No importa lo mucho que valoremos la intersubjetividad, siempre habrá cosas - información, sentimientos, experiencias, que elegimos no contar por nuestro propio deseo de privacidad y/o porque sospechamos que sería demasiado disruptivo, demasiado inquietante, demasiado dañino hacerlo de otra forma. Elegimos (parcialmente inconscientemente) lo que intentamos mantener en paréntesis o expresar basado en una mezcla de las necesidades, deseos y ansiedades nuestras y de nuestro paciente, junto con nuestras ideas clínicas y de lo que es terapéutico. $Y$ estoy convencida de que esto es cierto sin importar dónde nos coloquemos en el continuo restricción-expresividad.

Las teorías clínicas - como las posiciones políticas - casi siempre se formulan en oposición a otras contrastantes, una serie continua de correctivos que con frecuencia se convierten en oscilaciones pendulares. Los primeros escritos relacionales representaban un correctivo para los excesos de las teorías jerárquicas y unipersonales basadas en las pulsiones (Greenberg \& Mitchell, 1983). Mi trabajo sobre la contención representó un intento para 
re-equilibrar esa rectificación en una tercera dirección, detallando los límites de la mutualidad. Provocó su propia reacción, y en los '9o Tony Bass (1996) y yo tuvimos una animada discusión sobre la cuestión de si es posible contener, en lugar de retener o aferrarse; es decir, si contener meramente ofusca los 'elefantes en la habitación'. Con el tiempo, el pensamiento relacional ha continuado cambiando. Estamos más incorporados en los modelos de desarrollo que anteriormente (ver la detallada revisión de Seligman, 2003). De hecho, siguiendo la tendencia crítica al desarrollo - influido por Loewald, los teóricos del apego, Benjamin, y, quizás, mi propia posición - se enfocó de forma creciente en el papel de la dinámica relacional temprana y cómo esta informa la experiencia analítica tanto en el ámbito cognitivo como afectivo. Mientras que Mitchell nunca dio prioridad a las necesidades infantiles del paciente ni hablaba sobre la posibilidad de alcanzarlas simbólicamente, ya no insistía en el paciente-como-adulto. Cuando publicó Relacionalidad en 2000, articuló cuatro modelos interactivos a través de los cuales se organizan los patrones de conexión. Esta era la versión en capas de un adulto influido por una gama de modalidades relacionales, donde al menos algunas tienen su origen en la infancia. Esto subrayaba la multiplicidad de la experiencia del self y sugería que siempre hay formas en las que, de una vez o en rápida alternancia, el adulto se mueve entre los estados de adulto, niño y bebe.

Hoy nosotros los relacionales estamos en un territorio que es tan viejo como nuevo. Hemos rechazado modelos esquemáticos que se apoyan en la visión lineal del desarrollo. La noción de un proceso de crecimiento fijo y secuencial colisiona con Isa teorías del movimiento no-lineal y estados múltiples del self (Bromberg, 1991; Davies \& Frawley, 1994; Goldner, 1991; Corbett, 1993, 1998, 2006, 2008, 2011; Harris, 2009).

Sin embargo, las teorías de la multiplicidad (Mitchell, 1993) no niegan la posibilidad de experiencias de bebé dentro del proceso analítico, sino que le dan cabida. Porque los estados del self están en movimiento, no son unitarios; no tenemos que elegir entre bebé o adulto. Aun cuando nuestro paciente se siente como un adulto, tiene la capacidad quizás reconocida, quizás no - de acceder e incluso entrar temporalmente en un estado bebé. Y viceversa.

En la última década, otras tendencias relacionales, demasiadas para catalogar aqui, han entrado en la conversación sobre el desarrollo. Quizás el mayor eje central haya sido la contribución de la investigación sobre el apego y la teoría de sistemas dinámicos (ej. Ainsworth 1969; Hess \& Main, 2000; Stolorow, 1997; Beebe \& Lachmann, 1994; 2002). Discusiones sobre los procesos que subyacen a los distintos patrones de búsqueda de confort (ej. Hesse \& Main, 2000), junto con una exploración de los procesos interactivos de 
regulación mutua, completan nuestra comprensión de lo que hay detrás de la metáfora global de la contención y despliegan su dimensión no-verbal.

Los patrones de apego temprano se hacen sentir a través del tiempo incluso mientras se transforman. En este sentido, los investigadores infantiles invitaron a que los estados infantiles entraran de nuevo en la consulta y se dirigieron al legado del bebe, o incluso al bebé mismo, simultáneamente observando el complejo papel de la madre (y del analista) en la co-formación de estos patrones. Añadiría que aquí se implica también la infancia del analista; en ocasiones puede haber dos bebés en la consulta, y los patrones de regulación temprana se activan junto con - y en reacción a - los del paciente. Desde luego, este bebé (empírico) no es el vulnerable recipiente del cuidado materno suficientemente bueno - o no -de los teóricos de las relaciones de objeto. Es otro tipo de bebé, reactivo a y un participante en los tira-y-afloja propios de la madre. Aun, el legado de estos patrones tempranos, abren la puerta a la responsividad parental - es decir - analítica cuando los estados de malestar del paciente adulto incluyen la sombra de ese bebé.

Algunos de nosotros, los relacionales - incluida yo, Benjamin (1988; 1995), Davies (1994; 2004), Harris (2009), Bromberg (1998), Warshaw (1992) y Grand (2000) utilizamos modelos implícitos de desarrollo. Pero pensadores como Hoffman (2008; 2009) y Don Stern (1997) que no están interesados en los patrones de desarrollo sino en los patrones interactivos que siguen haciendo eco de algunos aspectos de estos temas.

La necesidad de momentos de responsividad sintonizada emergen a lo largo de nuestra vida, sin importar lo "separados" o reflexivos que seamos (esto lo aclara la idea de Ogden's 1986 de modalidades afectivas simultáneas pero cambiantes). Y no creo que esta necesidad esté limitada a pacientes con un historial de trauma temprano masivo; casi todos mis pacientes ( $y$ todos nosotros los analistas) tienen momentos cuando ese 'ya no bebé' es tan palpable como lo es nuestra identificación parental y fantasía reparadora. Como mi paciente mujer de negocios muy adulta que sintió que quería derretirse en mi sillón una fría tarde de noviembre, o mi paciente de Wall Street quien me telefoneó la semana pasada en pánico, por algo que su mujer había dicho y que hizo que le pareciera que el cielo se le caía encima. Recordó por primera vez en su vida que tenía a quien llamar: durante un momento me convertí en una presencia consoladora, alguien que podía recibir su desasosiego, aceptarlo en lugar de contradecirlo, sin des-regularme también. Se sintió contenido y lentamente se calmó lo suficiente y pudo pensar en cómo se estaba sintiendo. Juntos 'pusimos en escena' una versión de la metáfora parental. Pero sólo durante ese momento.

Lo que nos mete en líos es lo concreto: Cuando insistimos en que el paciente es un bebé, o cuando insistimos que es una adulta capaz de la mutualidad, corremos el riesgo de 
demandar una forma de falsa auto-complacencia. Nos saltamos la naturaleza interpenetrante de los estados de self bebé y adulto, y tiramos de uno $u$ otro de una forma que puede que nos parezca un 'como si' o pseudo. Cualquiera de estas pueden ser formas de avergonzar al paciente. El paciente visto como bebé puede sentir vergüenza sobre su envidia o su odio; el paciente visto como 'adulto' puede sentir vergüenza sobre su vulnerabilidad y sus deseos de fusión.

A lo largo de los años he ofrecido muchos ejemplos de trabajo dominados por la metáfora de la contención, organizado alrededor de estados afectivos centrales como la dependencia, la rabia, el narcisismo y la falta de consideración. He intentado ilustrar cómo la experiencia de reconocimiento consistente facilita el movimiento gradual hacia el intercambio intersubjetivo. Aquí describo un tratamiento en el cual los momentos de contención (organizado fuera del ámbito de la metáfora materna) no dominan, sino que enfatizan. A continuación, ofrezco algunas ideas para lo qué puede servir la función dinámica de la contención.

Mark, un académico al inico de sus 50 años, vino a análisis hace aproxima-damente una década. Se había criado con un padre despectivo, físicamente abusador, y una madre pasiva, mayormente ausente, que no parecía conectar mucho con él. La juventud de Mark se había caracterizado por ir a la deriva - de relación en relación y de carrera en carrera. Al llegar a mediana edad, Mark había conocido a su actual pareja Chris, y algo sobre la estabilidad uniforme de Chris reparaba a Mark lo suficiente como para asentarse en una relación y carrera razonablemente sólidas, aunque periódicamente asomaba su historial traumático. Al acudir a mi consulta, a instancia de Chris, Mark era defensivo y evitativo, pero también se lamentaba de ser consciente de que su irritabilidad estaba empañando su relación con Chris. Como él decía, "Chris me matará si no hago esto. Pero entonces, quizás simplemente me mate yo y a él primero. Hablando metafóricamente solamente, claro".

Sagaz y gracioso, Mark mantenía a raya una gran depresión y acordamos un tratamiento de tres sesiones por semana. Era auto-reflexivo de forma intelectualizada, oscilando entre estados de humor de enfado amargo, y un sentido del self más curioso y vivaz. Podía pensar sobre su pasado y conectarlo con su elección de pareja, alguien con quien era seguro enfadarse. Mark también notó que no había tenido allí una madre con quien enfadarse, bromeando que era bueno que yo tenía un impacto mayor que lo que mi tamaño podría sugerir. El fácil humor de Mark sería uno de los pilares de nuestro trabajo.

Pero la mayoría de las veces Mark no era gracioso; estaba dolorosamente triste y amargado. Escuchando sus reminiscencias, imaginaba la soledad y el miedo de este niño al lidiar con un poderoso e irritable padre y madre ausente. Ya que Mark hablaba tan 
libremente, no fui inmediatamente consciente de que todo iba bien solamente cuando yo sólo escuchaba. Cuando entraba en la conversación activamente -ya fuera para pregunta algo, comentar, o ofrecer una tentativa de interpretación, cuando expresaba mi sensación de lo que Mark estaba sintiendo o por qué podría estar diciendo algo - entonces no iba tan bien. Mark hacía más pausas breves y seguidamente continuaba hablando como si no me hubiera oído. Ocasionalmente asentía con la cabeza antes de continuar, pero sentía que este asentir con la cabeza era solo una forma de hacer que me callara. Cuando era particularmente persistente, Mark cambiaba el tema - normalmente a algo externo a ambos. Cuando Mark describía recuerdos especialmente dolorosos y yo reaccionaba verbalmente -por ejemplo diciendo - 'eso suena fatal', o incluso al hacer un sonido empático, pausaba solo brevemente antes de o bien hacer una broma o abandonar por completo el campo interior y lanzarse a una descripción de algo que estaba sucediendo en el exterior, en el mundo. Cuando le preguntaba a Mark si lo que había dicho le había molestado, ignoraba mi pregunta y a veces hacía otro chiste, pero siempre se iba a un tercer espacio. Mayormente ese tercer espacio tenia, que ver con el ámbito político y su sofisticado análisis del mismo. Aunque era consciente de su función defensiva, estaba absorta por la astuta (y resonante) perspectiva de Mark y me entretenían sus chistes.

Sin embargo también sabía que estábamos utilizando estas conversaciones como un modo de salir del estado del self consciente en el que Mark temía que caería (o ya había caído). Mark necesitaba mantenerse a distancia (entre nosotros) y de sí mismo, y mientras la ansiedad sobre la fusión probablemente subyacía bajo esta necesidad, parecía imposible nombrarla. Mucho se dejaba sin articular. Y así, cuando parecía que era el momento tan adecuado como cualquier otro, suavemente intenté nombrar algo de esto. Mark se quedó en el sofá muy quieto. Asintiendo con la cabeza, estaba intensamente sonrojado, pero se mantenía en silencio. Tras esperar un poco, dije aún más suavemente que pensaba que le había avergonzado mucho, que el ser 'visto' o comprendido por mi le hacía sentir dolorosamente expuesto. Tras una pausa, Mark asintió y dijo, casi en un susurro, "por favor no". Sintiendo que no podía decir más, solo dije "lo intentaré". Y así lo hice. Mayormente.

Mark toleraba conectar conmigo sólo cuando nuestro vinculo se mantenía superficial y en clave de humor. Yo luchaba para honrar esto (contenerle) dándole más espacio y reteniendo mis emociones, especialmente mi tristeza resonante con el dolor de Mark, un dolor que estaba casi siempre finamente velado por el humor. $Y$ nos adentramos en- 0 mejor, Mark se adentró- un tipo de auto-análisis del cual yo era más un testigo que un participante. 
Tuvo que pasar otro año completo antes de que Mark llorara en mi consulta y aun más tiempo hasta que se permitiera expresar el deseo, ni qué decir la necesidad de mi aportación, y más aún mi cuidado. Pero con el tiempo todo eso llegó, y gradualmente el "auto-análisis" de Mark se convirtió en un análisis diádico. Con el trabajo de una década a nuestras espaldas, estamos acercándonos a la compleción del trabajo, y recientemente hemos hablado sobre la idea de finalizar.

Aun así, la característica esquiva de Mark sigue siendo un hilo claro y presente. Sin embargo, ahora anuncia sus defensas intensificadas con una broma: "Ya vale de tus pensamientos, me voy a tomar un desvío a la izquierda", y alejándose de sí mismo, se adentra en la política de izquierdas. Listo, divertido, interesante, bromea conmigo y yo con él. Nos reímos y en ocasiones debatimos un poco. Es divertido para ambos.

He llegado a pensar sobre el humor de Mark, y mi respuesta apreciativa como algo que proporciona una función de contención co-creada, aunque sea atípica. Emerge cada vez que Mark toca los límites de su traumática historia o su potencial necesidad de mí, cuando su sentido de ser intacto se siente agudamente amenazado. Mark hace una rápida huida de ambos al mundo del humor, su versión del Show diario de John Stewart, un programa de televisión (de un comentarista político liberal) que nos encanta a ambos. Los chistes de Mark me hacen reír (mucho) y ayudan a re-equilibrar las cosas entre nosotros ya que igual que me ocurre a mi, él experiencia aspectos de su propia agencia y vivacidad mientras también de forma simbólica aviva a su madre depresiva y mortífera. El humor le contiene creando una amortiguación contra la doble amenaza de una exposición humillante (a la vista de un objeto que no le responde) y el ataque, ambos precipitantes de estados de aguda vergüenza.

Normalmente no conecto la contención con el humor; es un registro que se siente como más espontáneo, fácil, que representa la subjetividad de cada uno, un afecto tan interpenetrante. Utilizo la metáfora de la contención para describir el proceso mediante el cual intento nombrar y contener estados afectivos intensos como la ansiedad, la pena, rabia y desesperación. Al resonar con y aceptar una emoción o profundizar en la comprensión de mi paciente ("pero podrías experimentarlo, verlo de forma distinta"), se establece más espacio dentro del cual definir y elaborar la forma y los extremos de la emoción. A veces contener significa nombrar lo innombrable en el momento en que puede, apenas, ser soportado. O identificar, quizás amplificando, los aspectos inarticulados de una experiencia naciente, inarticulada o sólo parcialmente articulada (Slochower, 2004). A veces simbólicamente (a veces literalmente) extiendo mi mano en respuesta a un momento de desbordamiento afectivo, para contra-restar una dolorosa sensación de 
aislamiento o terror. O contengo a mi paciente en mi mente, llevando la memoria emocional de su estado afectivo entre nuestras sesiones.

Todo esto ocurrió en el tratamiento de Mark, así como también mucho trabajo que nada tenía que ver con la contención. Hice muchas interpretaciones y a veces le hablaba directamente (y de forma un poco provocativa, buscando la confrontación) a Mark sobre aspectos de mi (difícil) experiencia con él, sobre su irritabilidad y sarcasmo. También hubo "re-enactments", cuando le fallaba a Mark justo de la manera en que él necesitaba que no le fallara. Todo esto tuvo sus propios efectos terapéuticos - y contra-terapéuticos. Luchamos y también negociamos (Pizer, 2996). De hecho podría escribir un trabajo entero sobre los enactments y negociaciones en el tratamiento de Mark. Pero ya que aquí estoy pensando en los bebés, estoy inclinando todo al otro límite y subrayando el telón de fondo contra el que tuvieron lugar todas estas cosas más jugosas. Como el trasfondo de seguridad de Sandler (1960), nuestra risa fue el eje alrededor del cual se organizaba el resto. Aunque quizás algunos de vosotros diríais que los enactments eran el eje y la contención lo que mataba el tiempo entre enactment y enactment, como dijo Spezzano (1998).

La intensa vulnerabilidad de Mark a los estados de vergüenza hacía que fuera casi imposible nombrarlos ni explorarlos, sin embargo, acechaban los contornos de casi todo lo que decía. Y cuando eran evocados intensamente, eran intensamente des-estabilizantes. Sospecho que mi risa, a través de procesos de acción interpretativa (Ogden, 1994), ayudó a Mark para acceder y mantener un estado del self no-humillante justamente en los momento de vergüenza más aguda. Recientemente esto lo puso en palabras: "A veces pensaba que era un pelele patético y baboso. Alguien a quien todos señalan y de quien se mofan. Así que en cambio, conseguí que te rieras, y cuando lo hacías, re-encontraba otra parte de mí. Y ya no me sentía avergonzado." Sólo ahora, con el final a la vista, estamos explícitamente abriendo y trabajando con estas dinámicas de vergüenza. Parece probable que esta sea la última fase del trabajo que tenemos por realizar. Esencial pero evasivo fuera de la experiencia de la contención.

\section{Lo que nos llevamos}

Las metáforas de bebé y niño expresan la "realidad" fenomenológica de estos estados mientras temporalmente ignoran la otra realidad - la del paciente-como-adulto. Creo que podemos, finalmente, dar ambos por sentados.

Pero nunca han sido solamente los bebés los que necesitan ser contenidos. Los niños más mayores - y nosotros los adultos - a veces también lo necesitamos, a veces desde dentro 
de un estado del self más joven, y a veces desde uno muy adulto pero muy vulnerable. Anoche justamente, tuve una larga conversación con alguien en una crisis médica terriblemente atemorizante. En su propio lenguaje me dijo como se sentía contenida por la capacidad de los demás de encontrarse con ella emocionalmente, aunque no lo hubiera pedido. No hay nada infantil en esto. Sólo es humano.

Por tanto, ¿dónde estoy ahora? He descrito la contención de tantas formas, lo veo como algo que le da forma a tantos diferentes elementos terapéuticos, por lo que en este punto desecharía la palabra "C"3 por completo si pudiera. No es que no crea en la contención, sino que creo que hay algo demasiado esquemático y que con facilidad se le dan demasiadas interpretaciones al concepto. Se ha sangrado el concepto - en parte he sido yo quien la ha sangrado - más allá de sus límites originales y escucho cómo se utiliza para describir casi todo lo que nosotros los analistas hacemos, aparte de confrontar o realizar agudas interpretaciones. El concepto se utiliza con demasiada frecuencia para justificar o rápidamente categorizar lo que estamos haciendo y por qué 4 .

Sin embargo, como probablemente no podré deshacerme de la palabra, quiero ofrecer algunos pensamientos nuevos sobre sus funciones clínicas. Contener quiere decir ser testigo de la experiencia de mi paciente sin retarla, dando privilegio a su perspectiva de sí misma y permitiendo que se despliegue, recibida pero no alterada. Cuando contengo, actúo como testigo con una posición desde dentro. Este lugar emocional sirve como un amortiguador especialmente efectivo contra los estados de vergüenza (ver Orange, 2008; Morrison, 1989; Bromberg, 2009) porque suaviza el impacto y la alteridad del analista. La vergüenza está íntimamente asociada con ser visto desde fuera, ser 'mirado', y visto desde arriba5. Contener amortigua la vergüenza porque la experiencia de sintonía afectiva - de cualquier forma que se configure - crea un escudo contra la sensación de estar expuesto al ojo externo. Mi paciente y yo sentimos lo que ella siente juntas, y así llega a sentir conmigo y no vista por mí. Con el tiempo, podrá establecerse un "blindaje" que protege contra la humillación y que gradualmente nos permita entrar en el campo de la vergüenza. Juntas.

Se ha escrito sobre la función terapéutica de ser testigo en traumas graves (Laub, 1992; Grand, 2000,2010; Boulanger, 2008; Gerson, 2009; Harris, 2009, 2010; Rosenblum, 2010; Laub \& Auerhahn, 1993; Laub \& Podell, 1995). Esta literatura apoya el valor terapéutico de ser testigo en el trabajo con supervivientes del Holocausto y otras víctimas de lo innombrable. Sin embargo, creo que también es cierto que todos nuestros pacientes - y todos nosotros - hemos sido traumatizado en tanto en cuanto todos hemos tenido la experiencia del no-reconocimiento en momentos de aguda necesidad (Donnel Stern, 2009). 
Para muchos pacientes y a veces para el analista (Ruth Stein, 1997), la vergüenza está conectada con lo que se siente como una exposición de las necesidades infantiles. Sin embargo, para otros, la vergüenza se evoca a través de estados como la ira, el deseo, la codicia etc. Irónicamente, a veces es la experiencia misma de ser contenido lo que evoca la vergüenza. Imagino que no os sorprende escuchar que, en el contexto de nuestra tentativa exploración de la vergüenza, Mark en una ocasión dijo, 'necesito no necesitar que tu estés de ninguna forma en particular conmigo. Si siento tu apoyo me siento avergonzado del hecho de quererlo. Tiene que ser que tu estés bien con cómo estés. Y no lo es'. En ese punto de nuestro trabajo, no había forma de evitar la vergüenza.

La contención alude al elemento reparativo que se pone en escena ("enact"), como una forma de experiencia emocional correctiva. Cuando contenemos o cuando funcionamos como un "nuevo objeto" no lo definimos como correctivo en el sentido de Alexander (1950), pero estamos haciendo algo muy próximo a esto al ayudar a crear antídotos contra las experiencias interiorizadas de objetos toxicos, y así, a los estados de vergüenza (ver Cooper, 2007; Davies, 2008; Hoffman, 2008; 2009).

Por supuesto hacemos mucho más que esto y el resto de lo que hacemos también cuenta mucho. Identificar la dimensión de contención como figura o como base, depende de nuestra teoría. Pero la experiencia de la necesidad temprana permanece como una capa viva de la experiencia humana, y esto es donde la sombra del elemento de la contención entra en juego; nos guía a nivel de procedimiento con respecto a cuándo y cómo entramos en el dialogo clínico, cuan directamente y cuan profundamente.

La metáfora de desarrollo ha sido criticada por su idealización, tanto de la función analítica como terapéutica. Pero a mi me parece que incluso cuando formulamos el proceso terapéutico fuera de la idea de contener - ya sea si pensamos en la necesidad del paciente de la confrontación, autenticidad, mutualidad, experiencias de auto-objeto o reconocimiento - estamos idealizando algo.

Nuestro ideal representa nuestro deseo - y a menudo también nuestra necesidad - de curar, de cambiar, de entrar en conexión con, de hacer algo útil. Por supuesto, nuestra persona limita nuestra capacidad para cumplir ese ideal, y nos confronta con lo que he llamado la colisión psicoanalítica (Slochower, 2006). Las colisiones emergen, con independencia de nuestra alianza teórica, del espacio entre el ideal profesional al que aspiramos y la realidad de nuestra falibilidad humana.

Según escribía este trabajo, tuve que confrontar mi propia colisión: a pesar de mi inmersión en el tema de la contención, no trabajo con frecuencia como Winnicottiana. Normalmente soy bastante directa; esto es, intento ávidamente buscar una forma de articular lo que 
estoy pensando y 'me retengo' muy poco (ver Bass, 1996). De hecho, muchos de mis pacientes han señalado (a menudo de forma afectuosa pero no siempre), que apenas les parezco un analista que contenga; me describen con más frecuencia como alguien que 'llama las cosas por su nombre' aunque sí de forma amable. Incluso, mucho de mí está contenida dentro de la metáfora de la contención, reflejando las formas en las que intento contener.

Con el tiempo, he llegado a ser más expresiva de mi subjetividad, más relajada. Quizás menos cautelosa. Sin embargo, casi todo lo que hago a través de la exploración, confrontación y re-enactment, tiene lugar dentro de un entorno caracterizado por un conocimiento de fondo de la potencial necesidad de la contención, de la vulnerabilidad de mi paciente a experiencias de vergüenza. Por tanto, de alguna forma, incluso contengo cuando estoy empujando. Todo esto, por supuesto, se experimenta y expresa en un abanico de formas (buenas y malas) por distintos pacientes.

Por lo tanto, para volver al principio: Simplemente no hay ningún bebé - ni adulto - en la consulta, ya que ambos miembros de la díada se mueven de instante en instante, de forma imperceptible e inconsciente - hacia y alejándose de la relación con el otro como sujeto colaborativo. En este proceso, el paciente y analista contactan, ponen en escena y quizás cubren las expectativas de esos estados de bebé y niño, para bien o para mal.

No tenemos que abandonar la idea de un bebé psicoanalítico ya que puede de hecho, nadar en el agua de baño relacional - agua de baño que incluya un analista que contenga y que falle en la contención, que mayormente - pero no siempre - es capaz de ser un profesional reflexivo que tiene acceso a sus propios estados bebé y a veces 'los confunde' con los de su paciente (ver Seligman, 2003). La trayectoria del desarrollo, tal y como es, ha tenido tantos baches y reveses que sería absurdo llamarlo lineal. Aun así, la noción de la progresión de un mundo dominado por la experiencia de un solo sujeto, a uno caracterizado por subjetividades inter-penetrantes y la posibilidad de la mutualidad - en sí misma cambiante, más que una progresión lineal - sigue siendo atractiva. En el camino a este objetivo, creo que contenemos, cada uno en su forma idiosincrática. Pero ya que complicamos nuestra comprensión del lugar que la contención ocupa en nuestro trabajo, podríamos buscar otra palabra. ¿Alguien tiene alguna idea?

\section{REFERENCIAS}

Ainsworth, M.D.S. (1969). Object relations, dependency and attachment: A theoretical overview of the infant-mother relationship. Child Development. 40:969-1025. 
Alexander, F. (1950). Analysis of the therapeutic factors in psychoanalytic treatment. Psychoanal. Q. 19:482-500.

Aron, L. (1991). The patient's experience of the analyst's subjectivity. Psychoanal. Dial., 1:29-51.

Aron, L. (1992). Interpretation as expression of the analyst's subjectivity. Psychoanal. Dial. 2:475508.

Balint, M. (1968). The Basic Fault. London: Tavistock.

Bass, A. (1996). Holding, holding back, and holding on. Commentary on paper by Joyce Slochower. Psychoanal. Dial., 6:361-378.

Bass, A. (2001). It takes one to know one; or, whose unconscious is it anyway? Psychoanal. Dial., 11:683-702.

Bass, A. (2003). "E" Enactments in psychoanalysis: Another medium, another message. Psychoanal. Dial., 13:657-676.

Bassin, D. (1997). Beyond the he and she: Postoedipal transcendence of gender polarities. J. Amer. Psychoanal. Assn. Special Supplement: 1997.

Bassin, D., Honey, M. \& Kaplan, M. M. (eds.) (1994). Representations of Motherhood. New Haven, CT: Yale Univ. Press.

Bassin, D. (1999). Female Sexuality. Northvale, N.J.: Aronson.

Beebe, B. \& Lachmann, F. (1994). Representation and internalization in infancy: Three principles of salience. Psychoanal. Psych. 11:127-165.

Benjamin, J. (1986). A desire of one's own: Psychoanalytic feminism and intersubjective space. In Feminist Studies/Critical Studies. Ed. T. de Lauretis. Bloomington: Univ. of Indiana Press, 78-101.

Benjamin, J. (1988). The Bonds of Love: Psychoanalysis, Feminism and the Problem of Domination. New York: Pantheon

Benjamin, J. (1995). Like Subjects, Love Objects. New Haven: Yale Unversity Press.

Boulanger, G. (2008). Witness to reality: Working psychodynamically with survivors of terror. Psychoanal. Dial., 18: 638-657.

Bromberg, P.M. (1991). On knowing one's patient inside out: The aesthetics of unconscious communication. Psychoanal. Dial., 1:399-422.

Bromberg, P. M. (1998). Standing in the Spaces. Essays on Clinical Process, Trauma and Dissociation. Hillsdale, NJ: The Analytic Press.

Bromberg, P.M. (1993). Shadow and substance: A Relational perspective on clinical process. Psychoanal. Psych., 10:147-168.

Bromberg, P.M. (2006). Awakening the dreamer. Hillsdale, NJ: The Analytic Press.

Bromberg, P. (2011). In the shadow of the tsunami. Hillsdale, NJ: The Analytic Press.

Burke, W. F. (1992). Countertransference disclosure and the asymmetry/mutuality dilemma. Psychoanal. Dial., 2:241-271. 
Chodorow, N. (1978). The Reproduction of Mothering. Berkeley: Univ. of California Press.

Cooper, S.H. (2007). Alexander's corrective emotional experience: An objectivist turn in psychoanalytic authority and technique. Psychoanal. Q.: 76:1085-1102.

Corbett, K. (2008). Gender now. Psychoanal. Dial., 18:838-856.

Davies, J.M. \& Frawley, M.G. (1994). Treating Adult Survivor of Childhood Sexual Abuse: A Psychoanalytical Perspective. New York: Basic Books.

Davies, J.M. (1994). Love in the afternoon: A relational reconsideration of desire and dread in the countertransference. Psychoanal. Dial. 4:153-170.

Davies, J. M. (2004). Whose bad objects are we anyway? Repetition and our elusive love affair with evil. Psychoanal. Dial., 14:711-732.

Dimen, M. (1991). Deconstructing difference: Gender splitting and transitional space. Psychoanal. Dial. 1:335-353.

Dinnerstein, D. (1976). The Mermaid and the Minotaur. New York: Harper \& Row.

Epstein, L. (1987). The problem of the bad-analyst-feeling. Mod. Psychoanal. 12:35-45.

Fast, I. (1984). Gender Identity: A Differentiation Model. Hillsdale, NJ: The Analytic Press.

Gerson, S. (2009). When the Third is Dead: Memory, Mourning, and Witnessing in the Aftermath of the Holocaust. Int. J. Psychoanal. $90: 1341-1357$

Goldner, V. (1991). Toward a critical relational theory of gender. Psychoanal. Dial., 1: 249-272.

Grand, S. (2000). The Reproduction of Evil. Hillsdale, N.J.: The Analytic Press.

Grand, S. (2010). The Hero in the Mirror. New York: Routledge.

Greenberg, J. \& Mitchell, S. (1983). Object Relations in Psychoanalytic Theory. Cambridge, MA: Harvard Univ. Press.

Harris, A. (2009). "You must remember this. Psychoanal. Dial., 19:2-21.

Harris, A. (1991). Gender as contradiction. Psychoanal. Dial., 1: 197-224.

Harris, A. (2005). Gender as Soft Assembly. Hillsdale, N.J.: The Analytic Press

Harris, A. (1997). Beyond/outside gender dichotomies. Psychoanal. Dial., 7:363-366.

Harris. A. \& Botticelli, S. (2010). First, Do No Harm. New York: Routledge.

Hesse, E. \& Main, M. (2000). Disorganized infant, child, and adult attachment: Collapse in behavioral and attentional strategies. J. Amer. Psychoanal. Assn., 48: 1097-1128.

Hoffman, I. Z. (1991). Discussion: Toward a social-constructivist view of the psychoanalytic situation. Psychoanal.Dial., 1: 74-105.

Hoffman, I.Z. (1998). Ritual and Spontaneity in Psychoanalysis. Hillsdale, N.J.: The Analytic Press.

Hoffman. I.Z. (2008). Forging difference out of similarity: The multiplicity of corrective experience. Psychoanal. Q., 75:715-751. 
Hoffman, I.Z. (2009). Therapeutic passion in the countertransference. Psychoanal. Dial., 19: 617637.

Kraemer, S. (1996). "Betwixt the dark and the daylight" of maternal subjectivity: Meditations on the threshold. Psychoanal. Dial., 6:765-791.

Laub, D. (1992). Bearing witness: Or the vicissitudes of listening. In: Felman, S., Laub, D, Editors. Testimony: Crises of Witnessing in Literature, Psychoanalysis, and History, 57-74. New York: Routledge.

Laub, D., Auerhahn, N.C. (1993). Knowing and not knowing massive psychic trauma. Forms of traumatic memory. Int. J. Psycho-Anal., 74:287-302.

Laub, D. and Podell, D. (1995). Art and trauma. Int. J. Psycho-Anal. 76:995-1005.

Little, M. (1959). On basic unity. In Transference Neurosis and Transference Psychosis: Toward a Basic Unity, pp. 109-125. New York: Jason Aronson.

Mitchell, S. (1988). Relational Concepts in Psychoanalysis. Cambridge: Harvard University Press.

Mitchell, S. (1984). Object Relations Theories and the Developmental Tilt. Contemp. Psychoanal., 20:473-499.

Mitchell, S. (1988). Relational Concepts in Psychoanalysis. Cambridge, MA: Harvard Univ. Press.

Mitchell, S. (1993). Hope and Dread in Psychoanalysis. New York: Basic Books.

Mitchell, S. (1997). Influence and Autonomy in Psychoanalysis. Hillsdale, N.J.: The Analytic Press.

Mitchell, S. (2003). Relationality. Hillsdale, N.J.: The Analytic Press.

Modell, A. H. (1975). A narcissistic defence against affects and the illusion of self-sufficiency. Int. J. Psycho-anal., 56:275-282.

Morrison, A (1989). Shame: The Underside of Narcissism. Hillsdale, NJ: The Analytic Press.

Ogden, T. H. (1986). The Matrix of the Mind. New York: Jason Aronson.

Ogden, T. H. (1989). The Primitive Edge of Experience. New York. Jason Aronson.

Orange, D.M. (2008). Whose Shame is it Anyway? Contemp. Psychoanal., 44:83-100.

Pizer, S.A. (1998). Build Bridges. Hillsdale, NJ: The Analytic Press.

Renik, O. (1993). Analytic interaction: Conceptualizing technique in light of the analyst's irreducible subjectivity. Psychoanal. Q. 62:553-571.

Sandler, J. (1960). The background of safety. Internat.J.Psychoanal., 41:352-356.

Seligman, S. (2003). The Developmental Perspective in Relational Psychoanalysis. Contemp. Psychoanal., 39:477-508.

Slochower, J. (2010). Out of the analytic shadow: On the dynamics of commemorative ritual. Psychoanal. Dial. 21: 676-690.

Slochower, J. (2006). Psychoanalytic Collisions, Hillsdale, N.J.: Analytic Press. 
Slochower, J. (2004). But what do you want? The location of emotional experience. Contemp. Psychoanal., 40:577-602.

Slochower, J. (1999). Interior experience in analytic process. Psychoanal. Dial., 9: 789-809.

Slochower, J. (1996a) Holding and Psychoanalysis: A Relational Perspective. Hillsdale, N.J.: The Analytic Press.

Slochower, J. (1996b) Holding and the evolving maternal metaphor. Psychoanal. Rev., 83: 195-218.

Slochower, J. (1996c). The holding environment and the fate of the analyst's subjectivity. Psychoanal. Dial., 6:323-353.

Slochower, J. (1994). The evolution of object usage and the holding environment. Contemp. Psychoanal., 30: 135-151.

Slochower, J. (1993). Mourning and the holding function of shiva. Contemp. Psychoanal., 30:135151.

Slochower, J. (1992). A hateful borderline patient and the holding environment. Contemp. Psychoanal., 28: 72-88.

Slochower, J. (1991). Variations in the analytic holding environment. Int. J. Psycho-Anal., 72: 709-718.

Spezzano, C. (1998). Listening and interpreting-how relational analysts kill time between disclosures and enactments: Commentary on papers by Bromberg and by Greenberg. Psychoanal. Dial., 8:237-246.

Stein, R. (1997). Chapter 8 The Shame Experiences Of the Analyst. Progress in Self Psychology, 13:109-123.

Stern, D. (2009). Partners in Thought. New York: Routledge.

Stern, D. (1992). Commentary on constructivism in clinical psychoanalysis. Psychoanal. Dial., 3:331-364

Stern, D. (1997) Unformulated Experience: From Dissociation to Imagination in Psychoanalysis. Hillsdale, N.J.: The Analytic Press.

Stern, S. (1994). Needed relationships and repeated relationships: An integrated relational perspective. Psychoanal. Dial, 4:317-346.

Stolorow, R. D. (1997). Dynamic, dyadic, intersubjective systems: An evolving paradigm for psychoanalysis. Psychoanal. Psych., 14:337-346.

Tansey, M.J. \& Burke, W.F. (1989). From Projective Identification to Empathy. Hillsdale, N.J.: The Analytic Press.

Warshaw, S.C. (1992). Mutative Factors in Child Psychoanalysis: A comparison of Diverse Relational Perspectives in Skolnick, N. \& Warshaw, S.C. (Eds) Relational Perspectives in Psychoanalysis. Hillsdale,N.J.: The Analytic Press.

Winnicott, D. W. (1971). Playing and Reality. New York: Basic Books. 
Winnicott, D.W. (1963). Dependence in infant-care, in child-care, and in the psychoanalytic setting. In: The Maturational Processes and the Facilitating Environment. New York: International Universities Press, 1965 pp. 249-259.

Winnicott, D.W. (1960). The theory of the parent-infant relationship. In: The Maturational Processes and the Facilitating Environment. New York: International Universities Press, 1965 pp. 37-55.

Original recibido con fecha: 20/5/2018 Revisado: 30/6/2018 Aceptado: 28/10/2018

NOTAS:

\begin{abstract}
${ }^{1}$ Ver también el caso de Sarah (Slochower, 1996, p. 49-50). Involucrada en una poderosa necesidad para la resonancia emocional, Sarah hizo un paréntesis tras una dramática interrupción de nuestra sesión telefónica (fui gravemente arañada por un gato) y continuó como si nada hubiera ocurrido.

${ }^{2} \mathrm{~N}$. de T. La autora usa el término Holding, pero matiza que le parece más apropiado usar el término "Contención" incluyendo el sentido de la acepción "Sostenimiento"

${ }^{3} \mathrm{~N}$. de T.: "C", de contención

${ }^{4}$ Pero hay también en parte un punto débil. La idea de sostener es tan evocadora y romántica, que, al igual que muchos conceptos Winnicottianos, están muy manidos y otras veces es utilizada de forma muy concreta. Por tanto he oído a analistas describir cómo le han dado a un paciente un objeto transicional - como si alguien distinto al paciente pudiera imbuir lo concreto con la transicionalidad. En una ocasión un supervisado describió como 'sostuvo' a su paciente permaneciendo absolutamente imperturbable en respuesta a la foto de la nueva y bella novia del paciente. Yo diría que él estaba competitivamente reteniendo y utilizaba el concepto de la contención para racionalizar su comportamiento. De hecho, se puede justificar casi cualquier intervención como contención, apoyando una regresión, expandiendo el espacio transicional. Incluso el concepto de la regresión a la dependencia ha perdido su significado original como respuesta organizada a un analista capaz de recibir y contener estados afectivos intensos - necesidad/rabia temprana etc. - sin colapsar ni tomar represalias. La contención con frecuencia se mezcla con la noción de la "regresión" a un retorno a un modo anterior "menos maduro' de relacionarse, un anteproyecto de un tipo de proceso lineal en el cual reparamos al bebé.
\end{abstract}

${ }^{5}$ N. De T.: es decir, con desprecio. 